\title{
Perceptual mapping of practical ethics along the value chain: A multiple correspondence analysis with industry and cultural indices as supplementary variables.
}

\author{
by \\ Antonio Ladrón de Guevara \\ Universitat Pompeu Fabra \\ Economic \& Business Deparment \\ C/ Ramon Trias Fargas, 25-27 \\ 08005 Barcelona \\ Spain \\ Anna Torres \\ Universitat Pompeu Fabra \\ Economic \& Business Deparment \\ C/ Ramon Trias Fargas, 25-27 \\ 08005 Barcelona \\ Spain \\ \& \\ Josep A. Tribó ${ }^{1}$ \\ Universidad Carlos III de Madrid \\ Business Department \\ C/ Madrid 126B \\ (28903) Getafe \\ Spain
}

\footnotetext{
${ }^{1}$ The authors wish to thank Carlos Bendito (Triodos), Ramón Pueyo (Fundación Ecología y Desarrollo) and Sustainable Investment Research International Company (SiRi) for access to the SiRi 500 Global Profile database. We also acknowledge the financial support of the Ministerio de Ciencia y Tecnología, Grants, $N^{\circ}$. SEC2003-03797, SEC2001-0445 and SEJ 2004-00672.
} 


\title{
Perceptual mapping of practical ethics along the value chain: A multiple correspondence analysis with industry and cultural indices as supplementary variables.
}

\begin{abstract}
This paper presents findings from a study investigating a firm's ethical practices along the value chain. In so doing we attempt to better understand potential relationships between a firm's ethical stance with its customers and those of its suppliers within a supply chain and identify particular sectoral and cultural influences that might impinge on this. Drawing upon a database comprising of 667 industrial firms from 27 different countries, we found that ethical practices begin with the firm's relationship with its customers, the characteristics of which then influence the ethical stance with the firm's suppliers within the supply chain. Importantly, market structure along with some key cultural characteristics were also found to exert significant influence on the implementation of ethical policies in these firms.
\end{abstract}

Key words: Practical Ethics, Value Chain, Multiple Correspondence Analysis. 


\section{1/ Introduction}

Typically the literature on Business Ethics and Corporate Social Responsibility (CSR) has tended to describe the prioritizing of ethical actions and policies implemented by firms independently according to the different stakeholders involved, often relating these actions to industry-specific issues [see Wood (1991), Clackson (1995) and Carroll (1979, 1991) among others]. A recent study of leading FTSE companies conducted by Knox, Maklan and French (2005) for example, found that for most companies, building stronger ethical relationships with their stakeholders was mostly confined to their relationships with their customers, with a firm's CSR policy rarely extending to also include significant ethical dimensions in their relationships with their suppliers or other major stakeholders along the supply chain. Significantly, industry sector was also found to be a major driver of a firm's CSR policy.

From an instrumental stakeholder perspective however, one would suggest that good management implies building good relationships with a range of key stakeholders, especially within the supply chain and not simply confined to tho se with customers, which arguably should then provide important returns in terms of financial performance (Freeman, 1984; Waddock \& Graves, 1997). The basic assumption underpinning this perspective is that the CSR may be an important mechanism that should result in a far more efficient use of organizational resources (Orlitzky et al., 2003). It follows then that firms should try to define a CSR policy that integrates the different stakeholders along the value-generating chain, especially those that lay at the extreme points of the chain most notably customers and suppliers. In so doing value is added in the different stages along the supply chain which should then improve financial results (Jones, 1995; Hillman and Klein (2001).

Somewhat similar arguments can also be found mirrored within the Relational Marketing literature (e.g. Handfield and Bechtel (2002) or Coviello and Brodie (2001)) where the creation of new relational forms are advocated as a means of reinforcing the intangible asset of "trust" in relationships between buyers and sellers. It is suggested that once relationships rely on trust, firms cannot then bear the risk to become untrustworthy for some stakeholders for fear of damaging the trust developed with others. It would follow then that a firm should be able to send signals to its customers that it is trustworthy if it behaves ethically with its stakeholders that are at the other side of the supply chain, namely its suppliers. Our theory similarly conforms to this view. Firms typically prioritize customers' loyalty over suppliers. Yet, if firms want to send a powerful signal of ethical commitment with their customers, they should also behave ethically with their suppliers. 
Making use of Multiple Correspondence Analysis (MCA) techniques we have found the existence of an asymmetry in the distribution of the different sectors within a lattice representing both ethical dimensions - with customers and with suppliers-. This is indicative of the existence of an evolution of ethical policies beginning with customers and then becoming integrated in relationships with suppliers as times goes by.

Our analysis also reveals differences across industries in several ethic actions implemented along the supply chain. More specifically, Health Care, Consumer Discretionary, and Consumer Staple sectors display high levels of both consumer's and supplier's practical ethics, while Telecommunication, Financials and Utilities reveal strong asymmetries between both policies. We have found the former sectors to be those where customer loyalty is particularly important and firms may signal their ethical stance by being socially responsible with suppliers that are at the other side of the supply chain. Also, in these sectors, suppliers have market power suggesting that a firm is particularly aware to behave ethically with them in order to ensure the continued provision of their valuable resources.

Finally, we have also incorporated cultural factors in our overall analysis. According to Lu et al (1999), ethical decisions are related to two types of variables: situational and individual. Sector variables are situational (e.g. type of product or market position), while culture is representative of the individual set. Thus, we have made use of sector and cultural indicators to enrich our interpretation of the nature of ethical behaviors. Based on a similar approach used by Rawwas (2001), we have also applied Hofstede's cultural taxonomy in classifying consumer ethics and personality, and have identified "feminine" type of cultures, those relying on cooperation, as those associated firms that have more developed ethical policies with suppliers.

The rest of the article is structured as follows. In the next section we present the theoretical underpinnings. Next, we present the methodology and the empirical results. We then follow with a discussion of the results. The paper concludes with some key implications that should be of particular interest for managers.

\section{2/ Theoretical Underpinnings}

The new challenges of competition demand greater cooperation from the different stakeholders that participate in the supply chain. As pointed by Valenzuela and Sánchez (1999), this cooperation strategy does not look for the appropriation of added value but for added value generation. Hence, those stakeholders that are in the upstream of the supply chain (suppliers) should be in a close relationship with those in the downstream (customers), interchanging assets, human resources and information. At a last stage, suppliers "should 
be" like customers, adopt their point of view in order to provide pieces with the specific characteristics (e.g. quality certificates, delivery conditions, related services, etc) required by their customers.

A reverse marketing perspective (Blenkhorn \& Barting (1991) also points in this direction and emphasizes cooperative partnership between firms that buy and firms that supply different inputs, instead of the traditional adversarial attitude towards suppliers. This is justified in terms of investments in specific assets as well as to avoid uncertainty (acquire materials and/or technology). This relational marketing perspective is integrated in a total quality management view (Das et al., 2000) that advocates for customer satisfaction above all but recognizes the importance of developing trusting relationship with different stakeholders like suppliers (Monczka et al., 1998; Noordewier, 1989; Smith et al., 1995)). Remarkably the development of such an intangible asset like trust requires firms to adopt an integrative view in its ethical stance with the different stakeholders. It is not sustainable to be trustworthy in the relationship with one stakeholder (e.g. customer) and not to be trustworthy with another one (e.g. suppliers). Hence, there is a "natural" connection between the development of advanced marketing policies like relational marketing and the definition of an ethical policy that integrates the different stakeholders connected along the supply chain.

Summarizing, a firm aiming to stimulate efficient exchanges through the supply chain in an uncertain environment should behave ethically not only with customers but also with the stakeholders that are in the other side of the supply chain. Moreover, this kind of double ethical behavior allows for improvements in coordination (formal organizations, Blenkhorn \& Banting, 1991) and communication (informal organizations, Blenkhorn \& Banting, 1991) within the firm, as well as to establish the type of long-term relationship allowing for the development of intangible assets like trust (Handfield and Bechtel, 2002) that reduce uncertainty in the transactions among the stakeholders along the supply chain.

Our point is that the process that moves from an "adversarial" to an "obligational" (Morris and Imrie, 1993) relationship that considers suppliers as collaborators of a firm is gradual and starts from a firm's ethical stance with its customers. A maximizing firm, by backward induction, starts its ethical commitment with the stakeholders that are at the end of the supply chain and moves upwards to its suppliers. Once this is achieved, there is a feedback effect that reinforces the original commitment with customers. These latter consider more credible the ethical commitments of a firm that is able to behave ethically with the upstream stakeholders (suppliers). In this case, customers' demands will be more easily satisfied because a firm is able to demand specific requirements from their suppliers to satisfy customer interests. Hence, firms that want to maintain a strong long-term ethical 
commitment with its customers define an ethical policy departing from their customers and integrating ethical commitment with their suppliers at a later stage. This is a way to ensure from them the provision of the input required to satisfy the customers' demands. We can represent this dynamic with the following scheme 


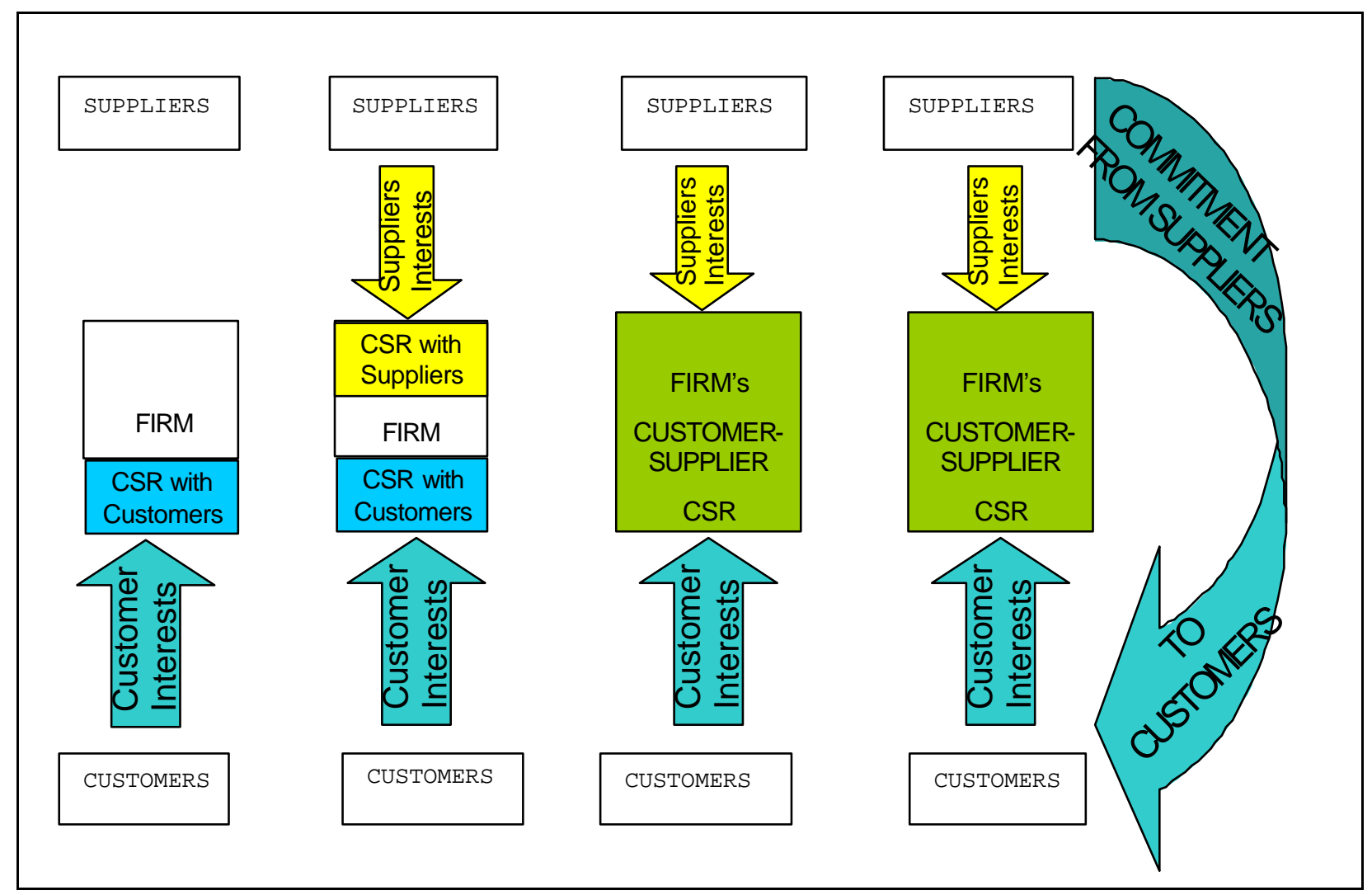

At a second level of analysis, the ethical stance of a firm depends on different factors that can be classified as: a) situational, and b) individual (Lu, et al., 1999). Among the situational factors, the sector is a major driver of firms' ethical policies. Firms that are competing in a market with high value-added products should be particularly aware of the interest of the stakeholders along the whole productive chain and, in particular, of their suppliers. Remarkably, in these types of value-added markets (e.g. automobiles, microcomputers), what is observed is the existence of a large number of customers and a restricted number of suppliers. According to Morris and Imrie1993) in these sectors, the change from the adversarial to the obligation contracting is particularly positive. Moreover, the lower the number of suppliers, the more powerful the suppliers and the more interested the buyers in getting trusting relationships with suppliers (Handfield and Bechtel, 2002). Thus, we should expect a relationship connecting sectors with high value-added products with a superior ethical stance towards suppliers.

Another aspect that it is worth considering is whether a firm's product is durable or not. This is relevant because customers of durable goods require a particular strong commitment from their sellers in order to provide high-quality post-sale services along the product lifetime. The quality of these services is closely related to the firm's ethical commitment with its customer. Furthermore, due to the aforementioned feedback effect, a possible way for a firm to signal strong ethical commitments with customers is to extend its 
ethical policies in order to incorporate its suppliers. Also, Coviello et al. (2002), connect the aforementioned relational marketing practices with consumer durable goods. We have related these practices with the development of trustworthy relationships as an enforcement mechanism to complete contracts. This will lead to ethical commitments with both customers and suppliers. Then, we should expect a superior ethical stance with suppliers in sectors with durable goods.

Among the individualism factors, we focus on culture. Culture is a fundamental determinant of ethical decision making ( $\mathrm{Lu}$ et al., 1999). In order to succeed in today's international markets, managers must recognize and understand how ideas, values and moral standards differ across cultures, and how these in turn influence marketing decision making (Laczniak, 1993). Importantly, the cultural values of a society are to a large extent the aggregation of the cultural values of the different stakeholders connected with the different firms of that society. Thus, societal cultural indices may be a good proxy of the type of cultural values within the firms of that society. According to Hofstede's cultural typologies, countries with small levels of PDI (power distances) combined with high levels of COL (collectivist cultures) are related with traits like the ability to cooperate and to sustain informal relationships with both superiors and group members. Contrarily, another combination like weak UAV (uncertainty avoidance) and high MAS (masculinity) implies behaviours emphasizing earnings, competitions, advancement and challenge. In these societies, economic growth is preferred to environment conservation. Thus, collaboration between the different stakeholders along the supply chain will be more difficult to be achieved in cultures relying on individualism or masculine-type cultures rather than in feminine-type ones. In these latter cultures, cooperation is in the roots of the society and it pays dividends for a firm to complement a customer ethical policy with a supplier ethical one. Hence, we expect a superior ethical stance towards suppliers in firms that are based on those cultures promoting cooperation values (feminine, collectivist-type with small levels of power distance) in comparison with those other cultures characterized by high levels of masculinity and individualism, or power distance.

\section{3/ Empirical Analysis}

\section{1/ Data}

Our sample is composed of 667 industrial firms of 27 different countries included in the 2002 SiRi Global Profile database, compiled by the Sustainable Investment Research International Company ( $\mathrm{SiRi}$ - the world's largest company specialized in the analysis of socially responsible investment based in Europe, North America, and Australia. The profile 
of each firm contains over 350 data points that cover all major stakeholder issues such as community involvement, environmental impact, customer policies, employment relations, human rights issues, activities in controversial areas (e.g. alcohol), supplier relations, and corporate governance. In this paper we focus on those items that relate a firm to its customers and suppliers. The specifics of the questionnaire are placed in the Appendix. Basically, the questions rate the relationship between a firm and the aforementioned two stakeholders taking into account the following dimensions: the degree of disclosure of the firm's commitment of good practices with these stakeholders; the importance and the specifics of the policy as well as the management of that policy that characterize the relationship with the implied stakeholders along the value chain and whether there are controversies with them.

We complement the previous database - that provides information of the sector (at 2-digit level from Gics) and the country of origin of the firms-, with cultural indexes elaborated according to Hofstede $(1979,1983)$. In particular, for each observation we have information related to power distance, masculinity, uncertainty avoidance and individualism.

\section{2/ Methodology and analysis.}

The methodology used in this analysis is multiple correspondence analysis (MCA). Correspondence analysis (CA) is an exploratory multivariate technique that converts a nonnegative data matrix of categorical variables into a particular type of graphical display in which the rows and columns are depicted as points (Greenacre \& Hastie, 1987). The joint graphical display obtained can help in detecting structural relationships among these variable categories.

Visualization of the association of a wide range of categorical variables is a strong advantage of this technique as well as the versatility that allows for applying this technique to a wide set of fields. Hoffman \& Franke (1986) survey papers where this technique has been applied to address different marketing problems. Applications to other fields like sociology, ecology or linguistics among others are not rare.

We are not going to use the standard way of CA, instead a generalization named multiple correspondence analysis (MCA). It is not the aim of this paper to provide a detailed explanation of MCA. Instead, we introduce the concepts required to interpret the maps (see the theoretical appendix for further clarifications). For more details see Greenacre (1984, 1991). 
Several standard software packages include MCA as a multivariate tool (i.e. SPSS or XIStat). For example, for the users of XIStat of SimCA, a simple correspondence analysis to the data described in the theoretical appendix should be run, with the adjustments in the results detailed in Greenacre (1984) (chap.18). On the other hand, SPSS contemplates the application of Homogeneity Analysis, which is mathematically equivalent to MCA (Greenacre, 1984) but directly offers the required statistics to interpret the maps.

Maps display principal coordinates for columns (Greenacre, 1984), in other words, the coordinates of the projected column points. We differentiate between columns of different nature. In one hand, the active points, corresponding to variables related with CSR customer and suppliers variables, and on the other hand the supplementary ones (cultural \& sector information). The supplementary variables (Greenacre, 1984) have the role of explanatory variables, which let to visualize clusters in terms of such information, instead of displaying firms, which could obscure the results since we have 667 observations. Then, even they do not contribute to the meaning of the principal axes, they appear displayed in the map to enrich the interpretation.

This particular way of coding the data allows us to interpret squared distances within each attribute -comparing different CSR practices related with customers/suppliers-, as well as between attributes -comparing CSR practices related with customers and suppliers, jointly with segmentation variables: culture \& industry- (Carroll, Green \& Schaffer, 1986). Put differently, close positions between two different points mean high levels of associations, while distant positions have to be understood as low levels of association.

\section{3/ Results}

\section{[Insert Figure 1]}

The map displays a horseshoe-like pattern (Greenacre, 1993). In particular, it indicates that the data are arranged along the first principal axis in an increasing gradient ranging from "low levels of practical ethics to the customers" to "high levels of practical ethics to the suppliers". As depicted in Figure 1, the variables corresponding to low levels of customer ethics are located on the top-left side. Then, moving along the horseshoe, higher levels of ethical practices oriented to customers are displayed, locating its maximum levels, around the centroid, with negative height with respect to the second principal axis. At this position, the supply-oriented ones just start with its lower levels, increasing as we move to the other extreme of the horseshoe effect, located on the top right of the map. This result 
reflects the strong asymmetry in the gradual implementation of ethic practices along the value chain in a cross-section analysis: firms usually start with customer-oriented actions, and as they evolve in practice, a supplier ethical perspective is then considered. This goes in line with related literature on CSR practices along the value chain (Carroll, 1991; Knox et al, 2005). Summarizing, this evidence seems to suggest that firms that want to promote its ethical stance start by behaving ethically with its customers and, as times goes by, they end up with an ethical behavior with is suppliers. This conforms to our theoretical arguments.

The second dimension, named as "dimension of polarization" (Greenacre, 1984), differentiates firms, which have a combination of extreme responses against those with middle responses that follow the general gradient along the horizontal axis.

To be more specific, we proceed describing the variables with higher squared correlation values (Greenacre, 1984) for the first two principal axes. To have a reference point, we should note that the maximum squared correlation value takes a value of $1^{2}$, while the minimum value, which implies no association at all, is equal to $1 / \mathrm{Q}$, being $\mathrm{Q}$ the number of variables (36). Focusing on the top-left position, we find the lowest levels of the customer reporting. This variable has a squared correlation value equals to 0,239 , with respect to the first principal axis, and 0,246, with respect to the second principal axis. Moving along the horseshoe, we find other advanced customer ethics, like Ch: customer happiness. Its squared correlation value with respect to the second principal axis is equal to 0,261 . In this direction, we get in a region where supplier ethics appear (e.g. variable Sc: content of suppliers reporting, with a contribution value of 0,3 for the first principal axis and 0,25 for the second principal axis). Finally, on the top-right corner we find the large levels of more advanced ethic variables, like Sch: Human Right Issues (more details in the appendix).

Once the dimensions have been explained in terms of the ethical variables, we add the information related to the supplementary - industry and culturat variables to complement such patterns. For this purpose, we present Figures $2 \mathrm{a}$ and $2 \mathrm{~b}$ where the set of variables located close to the centroid are displayed in a more clear scale.

[Insert Figures2a-b]

Consumer Staples, followed by Consumer Discretionary are the sectors that display the highest relationship with supplier's ethical practices (i.e. suppliers reporting, corporate

\footnotetext{
${ }^{2}$ Even the inherent bounds of the squared correlation value are between 0 and 1 , in practice the upper bound can be lower than 1 and the lower bound bigger than 0 . A value of 1 can only be attained in an artificial situation where the score of the particular firm is identical in all quantified categories. On the other hand, the lower bound to consider as reference point (no association at all) should be 1/Q, which corresponds to the average inertia for each principal axes (more details in Greenacre, 1991).
} 
statement and suppliers, and scope of suppliers' policy). Duran \& Sanchez (1999) point out that in these sectors firms are motivated to establish long-run relationships with the suppliers to achieve a sustainable competitive advantage in the market. Also, Pavitt (1984) shows that Consumer Discretionary and Consumer Staples display a high percentage in process innovation compared to product innovation (55\% versus $45 \%)$. The former type of innovation requires a closer relationship with the different stakeholders along the supply chain in comparison with the latter type of innovation. In Consumer Staple sector, suppliers can provide highly differentiable components, which add significant value to the final product. For instance, the joint research in packaging development (product innovation) or reduction in logistic costs (process innovation) is commonly given. These actions imply specific investments that will eventually increase the added value of the final product. What is relevant is that firms in these sectors, where the relationship with customers is more developed and the offered products present high value added, implement ethical policies with suppliers that we may interpret as a reinforcement mechanism to gain customers trust. This conforms to the ideas presented in the theoretical underpinnings.

Materials and Health Care sectors are allocated in the customer-oriented ethical region, characterized by high values of variables like customer satisfaction surveys and by lower levels of supplier's ethics. Energy is located close to them. Remarkably, this other cluster of sectors is mainly driven by product innovation activities (Pavitt, 1984) instead of process innovation. Consistently with what we have mentioned before, firms in sectors with relative lower process innovation activities, have relatively less incentives to implement ethical policies with suppliers and are more aware on developing ethical policies with customers. Unsurprisingly, there is an extensive literature related with CSR oriented just to customers in the Health care sectors: Health Care Equipment, Biotechnology \& Pharmaceuticals or Chemicals. For example, go to Dessing \& Flameling (2003).

Telecommunications sector, which is in the negative region with respect to the second principal axis, also displays high levels of customer ethics but almost non-existent ethics with respect to suppliers (i.e. located between "Sr3: the company does not disclose relevant information on suppliers issues, but it does communicate on that issue when requested and Ss2: suppliers' issues are not addressed in the corporate statement). The other sectors (like financial, transport services and industrial commercial services between others, display low levels in ethics practices along the value chain. These are industries where suppliers are easy to substitute and do not add significant value to the chain. As suggested by Duran \& Sanchez (1999) these competitive supply-market structures imply short-term visions with respect to the suppliers. Also, in these sectors the goods provided are not only of limited added value but intangibles products or services. According to our 
theory, this should be associated with less developed ethical policies with respect to the suppliers. The data confirms this prediction.

Even the majority of cultural indices are located close to the centroid still some associations are interpretable. We understand that such results are due to the fact that all the firms belonging to our sample are large firms. The main result, in agreement with Hofstede's typology appears in the combination given by low levels of masculinity (MAS1) and high levels of uncertainty avoidance (UAI2). These two indices appear on the right hand side of the centroid where supplier ethical policies are considered. Remember that such indices combination, according to Hofstede (1991), belongs to traits related with cooperative, friendly and emotional cultures. This result is also consistent with our theoretical discussion.

In MCA we took 1/Q as a reference point to consider which inertias are interpretable, where $\mathrm{Q}$ is the aforementioned number of active variables that play in the study. Since third and fourth principal inertias still have relevant information, we add a second map collecting this information. We hope to complement the information obtained in the previous map, getting different combinations of ethical practices explained by sector and cultural variables. In the first map we run the analysis and we realize that variable Ch1: There is no indication on the existence of customer satisfaction survey, is a strong determinant of the map and may distort the rest of the information. Then, we eliminate this level. The resulting map is depicted in figures $3 \mathrm{a}$ and $3 \mathrm{~b}$.

\section{[Insert Figure 3a-b]}

The map for the third and fourth principal inertias reveals information related to specific characteristics of the sectors included in the analysis. In one hand, the third principal axis groups sectors related to consumer markets (in the right hand side of the map), versus industrial markets (located on the left hand side). The most relevant information, in terms of ethical practices, appears on the fourth principal axis. This is driven by two specific ethical issues related to controversial situations due to the type of product and the market position. One extreme concentrates sectors that apply CSR to suppliers, while the other collects sectors that do not apply it. We have found that high positive values on this axis collect industries characterized by potential controversial marketing practices (i.e. Biotechnology \& Pharmaceuticals and Chemicals ${ }^{3}$ ). Such questionable practices are referred

\footnotetext{
3 These are subsectors that belong to the aforementioned Health Care and Materials sectors. For the overall sector, we had found less evolved suppliers' ethical policies (see Map 1) than for these subsectors. This is a
} 
to potentially unsafe products for the final consumer as highlighted in question Ct1 (with squared correlation value equal to 0,227$)$. Consistently with our theoretical underpinnings, we can explain this result because firms in such industries may try to signal their sensibility with regard to customers' interests by implementing ethical policies with suppliers. This will ensure from them the required commitment to satisfy customers' preferences. The counterpart of the previously described position aggregates firms benefiting from a high market power level over their clients (i.e. Telecommunications and Financial services). Question Cmp1 is capturing a market structure with no intermediaries, and where the clients face high transaction costs if they want to change (with a square correlation value of 0,287 ). Firms in these industries have fewer necessities to implement intensive ethical policies with regard to their customers. Thus, they do not need to use the ethical policy with suppliers as the aforementioned reinforcing mechanism to define such a policy. Moreover, we can expect that these firms will enjoy such a market power with customers if they can choose from a wide variety of different suppliers. In this case, consistently with our theory, firms do not need to implement ethical policies with suppliers.

Finally, regarding cultural indices, they are displayed close to the centroid, as was the case of the previous map. The most discriminative is collectivism, which appears on the right hand side of the map, close to the consumer markets that develop supplier and customer ethical practices. It goes in agreement with our theory.

\section{4/ Discussion and Conclusions}

In this paper we have explored the ethical policy followed by firms with regard to their different stakeholders inter-related along the supply chain. Also, at a second level of analysis, we have integrated sector and cultural factors in explaining different patterns of association of firm's ethical practices with regard to the different stakeholders, mainly customers and suppliers.

We have relied on Multiple Correspondence Analysis (MCA) to explore the relationship between the ethics with customer and suppliers. This technique allows for visualizing the association of a multiple battery of categorical variables and even inferring some dynamic connections between different relationships among these variables. In this research some sets of variables are included in an active way (customer and supplier ethical practices) while other sets, the explanatory ones, appear as supplementary ones (industry and cultural variables) (Greenacre, 1984). 
We have argued that firms' ethical policy should begin with an ethical commitment with customers and, in the most ethicaldeveloped sectors, suppliers should then be integrated into such ethical practices. This is a way to reinforce the initial ethical commitment with customers and ensure the satisfaction of their ethical claims. Consistently, in sectors where ethical policies with customers are particularly relevant (e.g. sectors with durable goods), we expect higher ratings in firms' ethical policies with suppliers. We also expect this result in those sectors where suppliers have market power (i.e. concentrated supplier markets). In these markets, firms are particularly aware to gain the suppliers trust. Finally, once we integrate cultural factors, our claim is that more developed ethical policies (those that integrate suppliers) should be present in "feminine-type" of cultures, based on Confucian and collectivist values.

We corroborate the previous theoretical statement applying the aforementioned MCA into a database composed of 667 industrial firms of 27 different countries with a wide dispersion of sectors and cultural indexes. Specifically, we have found the following results: first, for most industries, practical ethics are not simultaneously given for customers and suppliers. They start becoming customer focused, and only incorporate supplier-oriented ethical actions once when they have developed strong customer practices. The second main result reveals relevant sector-specific differences in ethics implementations due to differences in market structures. In particular, in those sectors where customer ethics should be strong (i.e. Consumer Staples and Consumer Discretionary), it is also observed an ethical commitment with suppliers. However, in those sectors where ethics with customers is less important (i.e. Financials and Utilities) and/or supply non-durable goods, the supplier component is not shown. Additionally, we have found low ratings in ethics with suppliers in those sectors where firms have large market power and, eventually can buy products from a wide set of suppliers. This conforms to our theoretical arguments. Finally, once we introduce cultural variables, we obtain that in feminine cultures, with high levels of uncertainty avoidance, firms are more supplier oriented than in masculine ones with low levels of uncertainty avoidance. Firms in this latter type of cultures are biased towards competition, advancement, or economic growth and they mainly focus on customer satisfaction.

\section{Managerial Implications}

From our paper we can extract some conclusions that may be of interest for managers:

First, managers of firms aiming to reinforce its ethical commitment with customer may well try invigorating its ethical stance with suppliers. This gives further credibility to 
the ethical stance with customers. Moreover, this two-tier ethical policy ensures the provision from the suppliers of the type of input that will satisfy the interest of customers.

Second, firms expanding their activities to sectors that are quite different from their primary sectors may well have to expand its ethical practices upwards in their supply chain and design ethical policies that also integrate their suppliers.

Third, managers of multinational firms that operate in different cultural environments should take into consideration the ethical values of each society. This means that they cannot directly translate the ethical practices towards suppliers implemented in the "masculine-type" cultural countries (i.e. anglo-saxon countries) to "feminine-type" cultural countries (i.e. asian countries). This should be taking into consideration by anglo saxonbased multinational firms that wants to enter in asian-emerging economies.

Last, managers should be aware that when they adopt relational marketing practices, the relationships with the different agents the firm is interacting with will not rely on formal contracts as time goes by. This means that the firm has to develop informal enforcement mechanism like trust with the different stakeholders in order to operationalize this relational marketing policy in the long-term. The definition of an integral ethical policy with different stakeholders -customers, suppliers- is a way of accomplishing such a trust in the long-term.

\section{Limitations and further research}

This paper has some limitations that are also opportunities for future research: First, we expect to conduct a proper dynamic analysis of a firm's ethical practices by using a panel data. This will allow us to investigate, among other things, whether the effect of cultural differences on the ethical policies followed by firms has been converging as time goes by. We plan to conduct such an analysis when we have access to a new wave of data provided by Siri. Second, our analysis is biased towards large firms. This has generated some perverse effects in the analysis of cultural variables. We have found that they are not very significant because of their limited variability in large firms. These firms are generally multinational that incorporate values of different cultures. Knox, Maklan and French (2005) also emphasize the size of the company as a determinant of CSR activities. Thus, exploring the differences in the ethical policy definition once we compare large and small firms may be of big interest. Last, it may be worth to conduct a micro econometric analysis of the effects on different variables like a firm's returns or stakeholders' loyalty that are generated by changes in a firm's ethical policy related to its different stakeholders. 


\section{Bibliography}

Blenkhorn, D. L. and Banting, P.M.: 1991, "How Reverse Marketing Changes Buyer-Seller Roles", Industrial Marketing Management 20, 185-191.

Carroll, A.: 1979, "A Three-Dimensional Model of Corporate Performance," Academy of Management Review 4(4), 497-505.

Carroll, A.: 1991, "The Pyramid of Corporate Social Responsibility: Towards the Moral Management of Organizational Stakeholders", Business Horizons, Jul-Aug, 39-48.

Carroll, J.D., P. E. Green and C.M. Schaffer: 1986, "Interpoint Distance Comparisons in Correspondence Analysis", Journal of Marketing Research Vol. XXIII (August), 271-80.

Clackson, M.:1995, "A Stakeholder Framework for Analyzing and Evaluating Corporate Social Performance," Academy of Management Review 20(1), 92-117.

Coviello, N. E.; Brodie, R. J.; Danaher, P. J. and Johnston, W. J.: 2002, “ How Firms Relate to Their Markets: An Empirical Examination of Contemporary Marketing Practices", Journal of Marketing 66 (3), 33-46.

Daboub, A. and J. Carlton: 2002, "Stakeholder Learning Dialogues: How to Preserve Ethical Responsibility in Networks," Journal of Business Ethics 41, 85-98.

Das A, Handfield R, Calantone R, Ghosh S.:2000, A contingent view of quality management - the impact of international competition on quality. Decis Sci J 31, 649-90.

Durán, J. L. and Sánchez, F.: 1999, 'The Relationships Between The Companies and Their Suppliers", Journal of Business Ethics 22, 273-280.

Franke, R. H., G. Hofstede and M. H. Bond: 1991, "Cultural Roots of Economic Performance: A Research Note”, Strategic Management Journal 12, 165-173.

Freeman, E. 1984. Strategic management: A stakeholder approach. Boston, MA: Pitman.

Greenacre, M.J.: 1991, "Interpreting Multiple Correspondence Analysis", Applied Stochastic Models and Data Analysis 7, 195-210.

Greenacre, M.J.: 1993, Correspondence analysis in practice. London: Academic Press.

Greenacre, M.J and Hastie, T.: 1987, "The Geometric Interpretation of Correspondence Analysis", Journal of the American Statistical Association 82 (398).

Handfield, R: B. and Bechtel, C.: 2002, "The role of trust and relationship structure in improving supply chain responsiveness", Industrial Marketing Management 31, 367-382.

Hair, J.F., Anderson, R.E., Tatham, R.L. \& Black, W.C.: 1998, Multivariate data analysis $\left(5^{\text {th }}\right.$ ed.). Upper Saddle River, NJ: Prentice-Hall. 
Hillman, A.J., \& Keim, G.D.: 2001. Shareholder value, stakeholder management, and social issues: What's the bottom line?, Strategic Management Journal 22, 125-139.

Hoffman, D. L. and Franke, G.R.: 1986, "Correspondence Analysis: Graphical Representation of Categorical Data in Marketing Research", Journal of Marketing Research, XXIII (August, 1986), 213-27.

Hofstede, G.: 1979, "Value systems in 40 countries: interpretation, validation, and consequences for theory", in Eckensberg, L.H., Lonner, W.J. and Poortinga, Y.H. (Eds), Cross-Cultural Contributions to Psychology, Swets \& Zeitlinger, Lisse, pp. 398-407.

Hofstede, G.: 1983, "The cultural relativity of organizational practices and theories", Journal of International Business Studies, Fall, 80-95.

Jones, T.M.: 1995. Instrumental stakeholder theory: A synthesis of ethics and economics. Academy of Management Review 20, 404-437

Knox, S., S. Maklan and P. French: 2005, "Corporate Social Responsibility: Exploring Stakeholder Relationships and Programme Reporting Across Leading FTSE Companies," Journal of Business Ethics 61, 7-28.

Levy: 1997, "Lean Production in an International Supply Chain," Sloan Management Review 38(2), 94-102.

Lu, L., Rose, G. M. and Blodgett, J. G.: 1999, "The Effects of Cultural Dimensions on Ethical Decision Making in Marketing: An Exploratory Study". Journal of Business Ethics $18,91-105$.

Mamic, I.: 2005, "Managing Global Supply Chain: The Sport Footwear, Apparel and Retail Sectors," Journal of Business Ethics 59, 81-100.

Monczka R, Peterson K, Handfield R, Ragatz G.: 1998, Determinants of successful vs. nonstrategic supplier alliances. Decis Sci 29, 553-77.

Morris, J. and R. Imrie: 1993, "Japanese Style Subcontracting - Its Impact on European Industries", Long Range Planning 26(4), 53-58.

Noordewier T.: 1989, "A comparison of blanket and systems contracts", J Purchasing Mater Manage 25, $34-42$.

Orlitzky, M., Schmidt, F.L., \& Rynes, S.L.: 2003, "Corporate social and financial performance: A meta-analysis", Organization Studies 24, 403-441.

Panapanaan, V., L. Linnanen, M. Karvonen and V. Tho Phan: 2003, "Roadmapping Corporate Social Responsibility in Finnish Companies," Journal of Business Ethics 44, 133-148. 
Payne, D. and C. Raiborn: 2001, "Sustainable Development: The Ethics Support the Economics,” Journal of Business Ethics 32, 157-168.

Rawwas, Mohammed Y.A.: 2001, "Culture, personality and morality", International Marketing Review 18, 2.

Smith K, Carroll S, Ashford S.: 1995, "Intra- and interorganizational cooperation: toward a research agenda", Acad Manage J 38, 7-23.

Waddock, S.A., \& Graves, S.B.: 1997 "The corporate social performance-financial performance link", Strategic Management Journal 18, 303-319.

Wood, D.: 1991, “Corporate Social Performance Revisited," Academy of Management Review 16(4), 691-718. 


\section{Figures}

Figure 1: Symmetric map. First principal axis inertia $(0,218)$ \& second principal axis inertia $(0,161)$.

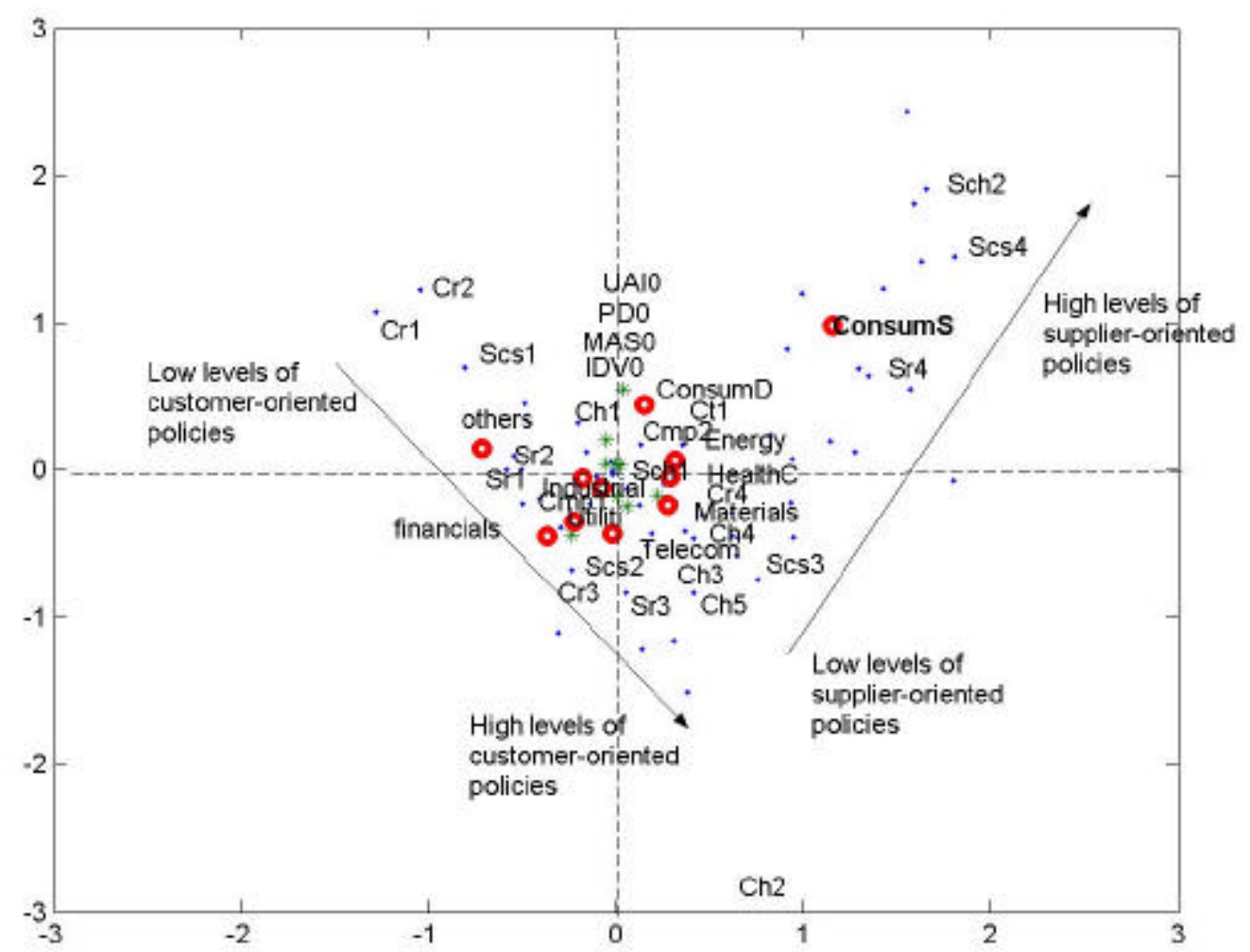

where

Sr: Suppliers reporting; Sc: Suppliers content; Scs: Suppliers corporate statement; Sp: Suppliers' policy; Ssp: Suppliers' scope; Si: Suppliers' initiatives; Sh: Suppliers human rights; Sch: Suppliers controversies on human rights, and

Cr: Customers reporting; Cc: Customer content; Ccs: Customers' statement; Cp: Customer Policy; Csp: Customer scope; Ch: Customer happiness surveys; $\mathrm{Cm}$ : Marketing procedures; Cs: Customer happiness; Ci: Customer initiatives; Ct: Customer controversial; Cmp: Customer power.

A rating scale is used to collects the different degrees in the application of each ethical practices. The lowest level is captured with number 1. The upper bound varies according to the number of levels of each particular variable. See the appendix for more detail.

UAI1: Low uncertainty avoidance; UAI2: High uncertainty avoidance; UAI0: Missing value on this variable. MAS1: Feminine culture; MAS2: Masculine culture; MAS0: Missing value on this variable. IDV1: Collectivism culture; IDV2: Individualism culture; IDV0: Missing value on this variable. PD1: Low power distance; PD2: High power distance; PD0: Missing value on this variable.

The notation appears more detailed in the appendix. 
Figure 2a: (Rescaled) Symmetric map. First principal axis inertia $(0,218)$ \& second principal axis inertia $(0,161)$

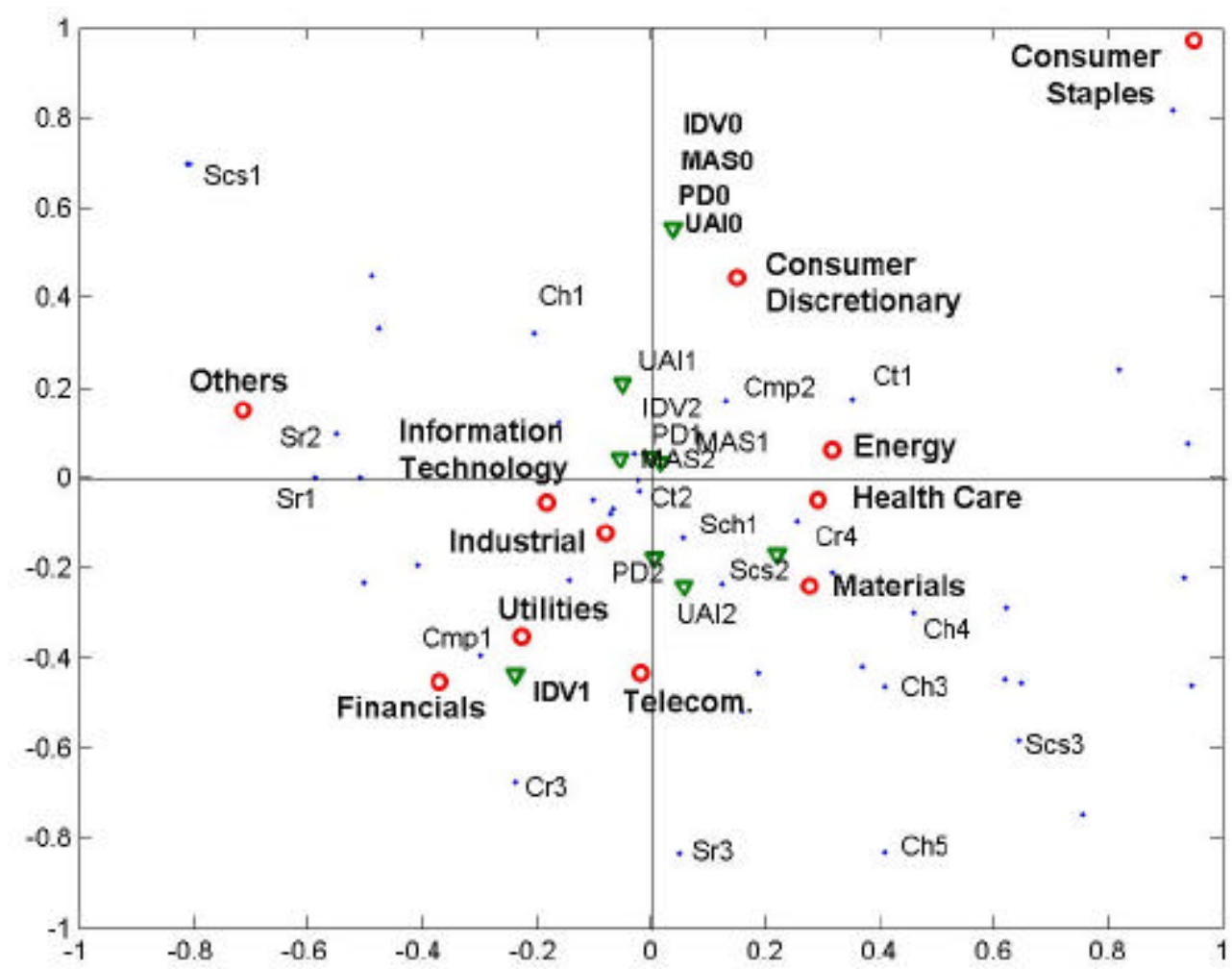

where

Sr: Suppliers reporting; Sc: Suppliers content; Scs: Suppliers corporate statement; Sp: Suppliers' policy; Ssp: Suppliers' scope; Si: Suppliers' initiatives; Sh: Suppliers human rights; Sch: Suppliers controversies on human rights, and

Cr: Customers reporting; Cc: Customer content; Ccs: Customers'statement; Cp: Customer Policy; Csp: Customer scope; Ch: Customer happiness surveys; Cm: Marketing procedures; Cs: Customer happiness; Ci: Customer initiatives; Ct: Customer controversial; Cmp: Customer power.

UAI1: Low uncertainty avoidance; UAI2: High uncertainty avoidance; UAI0: Missing value on this variable. MAS1: Feminine culture; MAS2: Masculine culture; MAS0: Missing value on this variable. IDV1: Collectivism culture; IDV2: Individualism culture; IDV0: Missing value on this variable. PD1: Low power distance; PD2: High power distance; PD0: Missing value on this variable. 
Figure 2b: (Rescaled) Symmetric map of industries. First principal axis inertia $(0,218)$ \& second principal axis inertia $(0,161)$

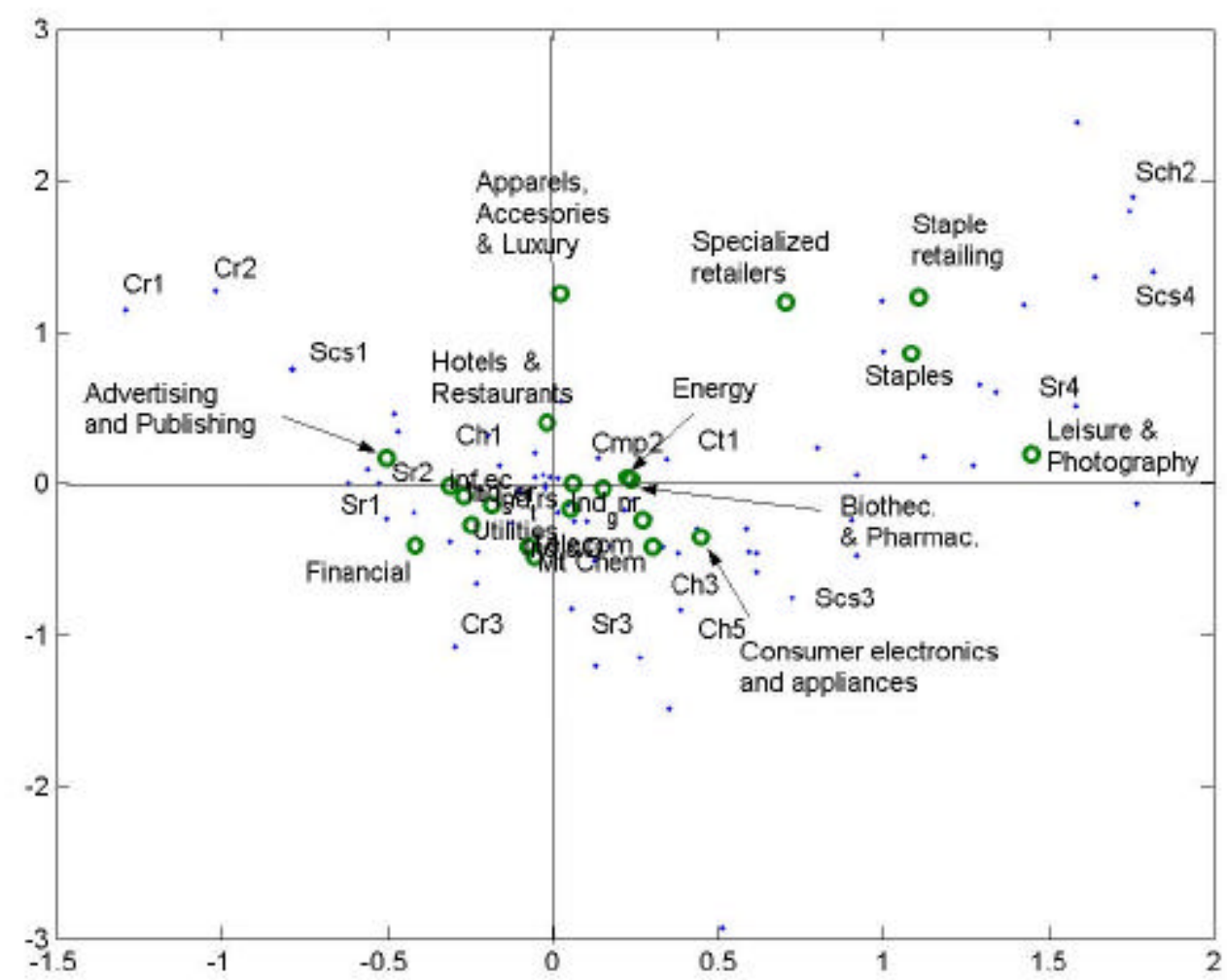

where

Sr: Suppliers reporting; Sc: Suppliers content; Scs: Suppliers corporate statement; Sp: Suppliers' policy; Ssp: Suppliers' scope; Si: Suppliers' initiatives; Sh: Suppliers human rights; Sch: Suppliers controversies on human rights, and

Cr: Customers reporting; Cc: Customer content; Ccs: Customers' statement; Cp: Customer Policy; Csp: Customer scope; Ch: Customer happiness surveys; $\mathrm{Cm}$ : Marketing procedures; Cs: Customer happiness; Ci: Customer initiatives; Ct: Customer controversial; Cmp: Customer power. 
Figure 3a: Symmetric map. Third principal axis inertia $(0,136)$ \& fourth principal axis inertia $(0,108)$.

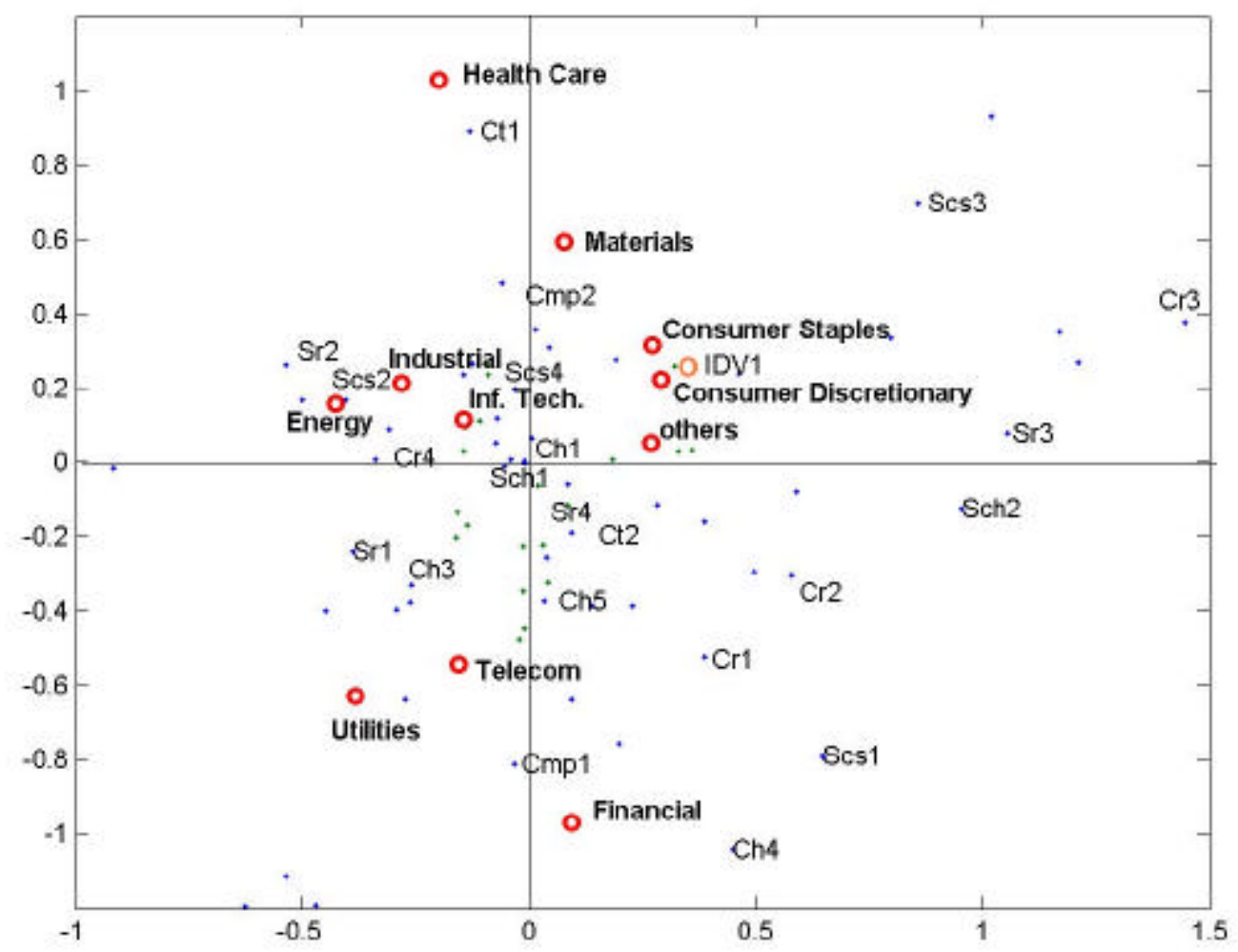

where

Sr: Suppliers reporting; Sc: Suppliers content; Scs: Suppliers corporate statement; Sp: Suppliers' policy; Ssp: Suppliers' scope; Si: Suppliers' initiatives; Sh: Suppliers human rights; Sch: Suppliers controversies on human rights, and

Cr: Customers reporting; Cc: Customer content; Ccs: Customers' statement; Cp: Customer Policy; Csp: Customer scope; Ch: Customer happiness surveys; $\mathrm{Cm}$ : Marketing procedures; Cs: Customer happiness; Ci: Customer initiatives; Ct: Customer controversial; Cmp: Customer power.

IDV1: Collectivism culture 
Figure 3b: Symmetric map of industries. Third principal axis inertia $(0,136) \&$ fourth principal axis inertia $(0,108)$.

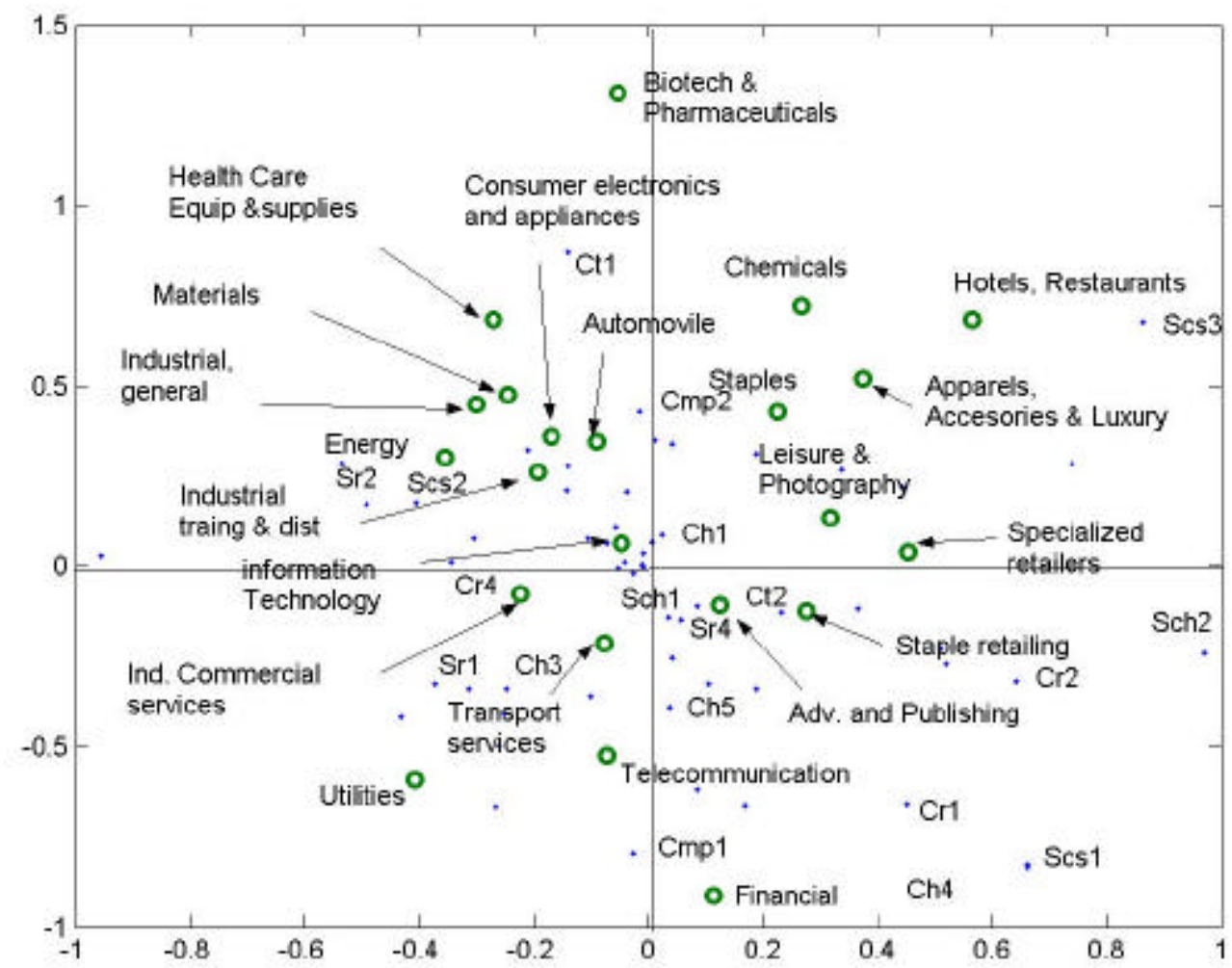

where

Sr: Suppliers reporting; Sc: Suppliers content; Scs: Suppliers corporate statement; Sp: Suppliers' policy; Ssp: Suppliers' scope; Si: Suppliers' initiatives; Sh: Suppliers human rights; Sch: Suppliers controversies on human rights, and

Cr: Customers reporting; Cc: Customer content; Ccs: Customers' statement; Cp: Customer Policy; Csp: Customer scope; Ch: Customer happiness surveys; $\mathrm{Cm}$ : Marketing procedures; Cs: Customer happiness; Ci: Customer initiatives; Ct: Customer controversial; Cmp: Customer power. 


\section{Empirical Appendix}

I) Description of CSR variables:

Suppliers.

\section{Sr: Suppliers reporting.}

Sr4: The company discloses relevant information on its relations with suppliers.

$\mathrm{Sr} 3$ : The company does not disclose relevant information on suppliers issues, but it does communicate on that issue when requested.

Sr2: The company does not disclose relevant information on suppliers issues and does not communicate information on that issue when requested.

Sr1: The company was not contacted yet.

\section{Sc: Content of suppliers reporting (supplier content)}

Sc7: The company publishes very comprehensive and in-depth information on suppliers.

Sc6: The company publishes comprehensive and in depth information on suppliers

Sc5: The company publishes superficial information on suppliers.

Sc4: The company publishes very superficial information on suppliers.

Sc3: the company clearly states not to address the issue.

Sc2: It is not possible to assess the content of the reporting.

Sc1: Not relevant (because there is not disclosure)

\section{Scs: Corporate statement and suppliers (supplier corporate statement)}

Scs4: Suppliers' issues are addressed in the corporate statement.

Scs3: Suppliers' issues are addressed vaguely in the corporate statement.

Scs2: Suppliers' issues are not addressed in the corporate statement.

Scs1: There is no visible corporate statement.

\section{Sp: Suppliers' policy}

Sp3: The company has one or more policies (or section of its business cod), which constitute a specific suppliers' policy.

Sp2: The company does not have a specific suppliers' policy.

$\mathrm{Sp1}$ : There is no indication on the existence of a specific suppliers policy.

\section{Ssp: Scope of suppliers' policy (suppliers' scope)}

Ssp6: The principles are precise guidelines addressing a majority of suppliers' issues.

Ssp5: The principles are general guidelines addressing a majority of the suppliers' issues.

Ssp4: The principles address a minority of the suppliers' issues.

Ssp3: It is not possible to assess the content of the principles.

Ssp2: The company has a general suppliers' policy, which does not address any of the issues.

Ssp1: Not relevant (because there is no policy).

\section{Si: Suppliers' initiatives}

Si3: The company has already taken one or several relevant initiatives expressing its commitment for its suppliers.

Si2: There is neither, visible initiatives or controversies.

Sil: The company faces controversies related to its relationship with suppliers. 
Sh: Exposure to human rights issues in supply chain (suppliers human rights)

Sh3: The company does not rely heavily on vendors or contractors, because it is active in services, software. Hi-tech or because it is vertically integrated.

Sh2: The company is active in the production, wholesaling or retailing of manufacture goods (electronics, cars, etc.).

Sh1: The company is active in the production, wholesaling or retailing of the following items (foods, d-I-y, furniture, sporting goods, clothes, toys, plants).

\section{Sch: Human rights issues (suppliers controversies on human rights)}

Sch2: existence of controversies over human rights issues in the supply chain.

Sch1: There is no indication on the existence of such controversies.

\section{So: Other issues (suppliers other controversies)}

So2: Existence of controversies over relationship with suppliers.

So1: There is no indication on the existence of such controversies.

Customers

\section{Cr: Customers reporting}

$\mathrm{Cr} 4:$ The company discloses relevant information on its relations with its customers.

$\mathrm{Cr} 3$ : The company does not disclose relevant information on customer issues, but it does communicate on that issue when requested.

$\mathrm{Cr} 2$ : The company does not disclose relevant information on customer issues and does not communicate information on that issue when requested.

$\mathrm{Cr} 1$ : The company was not contacted yet.

\section{Cc: Content of customer reporting (customer content).}

Cc5: The company publishes I-depth and comprehensive information on customers.

$\mathrm{Cc} 4$ : The company publishes superficial information on customers.

Cc3: The company clearly states not to address the issue.

$\mathrm{Cc} 2$ : It is not possible to assess the content of reporting.

$\mathrm{Cc1}$ : Not relevant (because there is no disclosure).

\section{Ces: Corporate statement (customers' statement)}

Ccs4: Customers' issues are addressed in the corporate statement.

Ccs3: Customers' issues are addressed vaguely in the corporate statement.

Ccs2: Customers' issues are not addressed in the corporate statement.

Ccs1: There is not visible corporate statement.

\section{Cp: Customer Policy.}

Cp3: The company has one or more policies (or section of its business code) which constitute a specific customer policy.

$\mathrm{Cp} 2$ : The company does not have a specific customer policy.

$\mathrm{Cp} 1$ : There is no indication on the existence of a specific customer policy.

\section{Csp: Scope of customer policy (customer scope)}

Csp5: The principles address a majority of relevant issues.

Csp4: The principles address a minority of relevant issues.

Csp3: The principles just mention quality and/or customer focus.

Csp2: It is not possible to assess the content of the principles.

Csp1: Not relevant (because there is no customer policy). 


\section{Ch: Customer satisfaction surveys_(customer happiness surveys)}

Ch5: Comprehensive customer satisfaction surveys are conducted regularly.

Ch4: Customer satisfaction surveys are conducted, but not on a regular basis or not comprehensive.

Ch3: It is not possible to assess the frequency of customer satisfaction surveys.

Ch2: Customer satisfaction surveys are not conducted.

Ch1: There is no indication on the existence of customer satisfaction surveys.

\section{Cm: Marketing procedures.}

$\mathrm{Cm} 5$ : The company has relevant guidelines/procedures on marketing issues (advertising guidelines, selling methods, etc.).

$\mathrm{Cm} 4:$ The company has vague marketing procedures.

$\mathrm{Cm} 3$ : It is not possible to assess the marketing procedures.

$\mathrm{Cm} 2$ : The company does not have marketing guidelines/procedures.

$\mathrm{Cm} 1$ : There is no indication on the existence of marketing guidelines/procedures.

\section{Cs: Customer satisfaction (customer happiness)}

Cs4: The results of the customer satisfaction surveys express a high satisfaction.

Cs3: The results of the customer satisfaction surveys express a satisfaction in the average.

Cs2: The results of the customer satisfaction surveys express a low satisfaction.

Cs1: There is no information on customer satisfaction.

\section{Ci: Customer initiatives.}

Ci2: The company has already taken one or several relevant initiatives expressing its commitment to customers.

Ci1: There is no indication on the existence of this kind of initiatives.

\section{Ct: Type of products (customer controversial)}

$\mathrm{Ct} 2$ : The marketing of the type of products offered by the company is not controversial.

$\mathrm{Ct} 1$ : The marketing of the type of products offered by the company may be controversial (the type of product is potentially unsafe for the consumer).

\section{Cmp: Market position (customer power)}

Cmp2: The company has not market power over its clients.

Cmp1: The company benefits form market power over its clients either because of the lack of competitors, or because the client faces high transaction costs if it want to change).

II) Cultural indexes:

UAI1: Low uncertainty avoidance (value below 50\% according to Hofstede's uncertainty avoidance index, 1991)

UAI2: High uncertainty avoidance (value above $50 \%$ according to Hofstede's uncertainty avoidance index, 1991)

UAI0: Missing value on this variable

MAS1: Feminine culture (value below 50\% according to Hofstede's masculinity index, 1991)

MAS2: Masculine culture (value above 50\& according to Hofstede's masculinity index, 1991)

MAS0: Missing value on this variable 
IDV1: Collectivism culture (value below 50\% according to Hofstede's individualism index, 1991).

IDV2: Individualism culture (value above 50\% according to Hofstede's individualism index, 1991).

IDV0: Missing value on this variable.

PD1: Low power distance (value below 50\% according to Hofstede's power distance index, 1991).

PD2: High power distance (value above 50\% according to Hofstede's power distance index, 1991).

PD0: Missing value on this variable.

III) Sectors

\section{Consumer Discretionary:}

a) Automobile manufacturers + auto parts \& equipment + tires \& rubber

b) Apparel, accessories \& luxury goods

c) Consumer electronics + home furnishings + homebuilding + household appliances

d) Leisure products + photographic products

e) Hotels, resorts \& cruise lines + restaurants + casinos $\&$ gaming

f) Advertising + broadcasting $\&$ cable TV + movies $\&$ entertainment + publishing

g) Apparel retail + catalog retail+ department stores + distributors + general merchandise stores + home improvement retail + specialty stores

\section{Consumer staples}

Energy

h) Retailing

i) Others

\section{Financials}

Health care

j) H.C. equipment + H.C. suppliers

k) Biotechnology + Pharmaceutical

\section{Industrials}

1) Aerospace $\&$ defense + building products + construction $\&$ engineering + construction \& farm machinery \& heavy trucks +Electrical components \& equipment + Industrial conglomerates + Industrial machinery

m) Treading companies $\&$ distributors

n) Diversified commercial services + Employment services

o) Air freight $\&$ logistics + Airlines + airport services + highway \& railtracks + marine + marine ports $\&$ services + railroads.

\section{Information technology}

\section{Materials}

p) Commodity chemicals + diversified chemicals + fertilizers and agricultural chemicals + specialty chemicals

q) Others

\section{Telecommunication Services}

\section{Utilities.}




\section{Theoretical Appendix}

The main difference between CA and MCA relies in the number of discrete variables involved. While simple correspondence analysis is limited to analyse categories coming from two variables, MCA does not have this restriction The data matrix in this case is an indicator matrix (with dummy variables) expressed in the form of cases-by-variables. In our example, the cases are the particular firms and variables include ethical practices related with suppliers and customers, as well as, cultural index, related with the country of origin, and sectors where firms belong. The structure of the data matrix is the following: $\mathbf{Z}(I x J)=$ $\left[Z_{1} \ldots Z_{Q}\right]$, where the $q$ th variable has $j_{q}$ categories and hence $Z_{q}$ is $I x J_{q}$ and that $J=\sum_{q=1}^{Q} J_{q}$ is the total number of categories. Then, we have $J_{1} x \ldots x J_{q}$ types of different possible responses. For example, in our application, in the set of CSR to the suppliers, one question is: h21: suppliers reporting. This variable has 4 levels, then, $J_{\text {Supplierers Re porting }}=4$.

It is not the aim of this paper to provide with a detailed explanation of MCA. Instead, we introduce the concepts required to interpret the maps. For more details go to Greenacre (1984). 